\section{P1-51 COMPARISON OF FIVE, WITH AND WITHOUT TIME- DEPENDENT COVARIATES, SURVIVAL MODELS AS DESCRIPTORS FOR CULLING DISTRIBUTIONS IN DAIRY COWS}

doi:10.1136/jech.2011.142976c.44

${ }^{1} \mathrm{H}$ Sharifi, ${ }^{*} \mathrm{~A}$ R Bahonar, ${ }^{3} \mathrm{~A}$ Sharifi, ${ }^{4} \mathrm{P}$ Kostoulas. ${ }^{1}$ Department of Food Hygiene and Public Health, Faculty of Veterinary Medicine, Shahid Bahonar University of Kerman, Kerman, Iran; ${ }^{2}$ Department of Food Hygine and Quality Control, Faculty of Veterinary Medicine, University of Tehran, Tehran, Iran; ${ }^{3}$ Department of Ophthalmology, Kerman University of Medical Science, Kerman, Iran; ${ }^{4}$ Laboratory of Epidemiology, Biostatistics and Animal Health Economics, University of Thessaly, Karditsa, Greece

Introduction This paper presents the results of a study to evaluate survival analysis the effect of treated diseases on the culling rate (remove from herd) in dairy cows.

Methods Five different models, with and without time-dependent covariates, using Gompertz distribution were studied. Model 1 treated diseases as a binary and time-independent covariates. Models 2 through 5 treated diseases as time-dependent covariates. For each observation, we split follow-up time in intervals each corresponding to a different lactation month. In other words, each observation from study entry until culling or censoring was split into several one-month observations by Lexis expansion of the original dataset. Model 2 assumed an animal experience a certain disease from the beginning of the occurrence of that disease by the end of follow-up period. Model 3 assumed cows are at risk from the begging of the study until the disease occurred (inverse of model 2). In model 4 and 5 an animal was assumed to experience a certain disease for 1 month if the disease occurred during this period. The only difference is in model 4 assumed diseases occurred only one time and in model 5 multiple disease occurrences at different months were considered as different episodes.

Results According to Akaike's Information Criterion (AIC) value and Cox-Snell residuals model 5 was the best model.

Conclusion A comparison of culling models with and without timedependent covariates found that models without time dependency tended to seriously underestimate the risk of a disease on culling.

\section{P1-52 THE IMPLICATIONS OF USING DIFFERENT METHODS TO MEASURE ETHNICITY IN A COHORT STUDY}

doi:10.1136/jech.2011.142976c.45

S Simmonds, D Sarfati, ${ }^{*}$ R Harris, G Purdie. Otago University, Wellington, New Zealand

Introduction Maori, the indigenous population of New Zealand, have the right to good health, healthy conditions and high quality epidemiological data. Consistent, comprehensive ethnicity data are crucial for appropriate representation of Maori health status and in order to monitor governmental progress towards equity in health. Maori have been undercounted in health datasets in the past and different methods of adjusting for this undercount have been developed and used in the calculation of population rates. This study investigates the implications of using four different methods to measure ethnicity in a cohort study.

Methods Using a cohort of patients with ischaemic heart disease (IHD), a sensitivity analysis was applied to determine the impact of four different methods of measuring Maori ethnicity on outcomes that determine disparities in both mortality and receipt of procedures, between Maori and non-Maori with IHD.

Results There was some slight variation in results with the use of different methods to measure ethnicity. Overall however, the interpretation of the results would remain largely unchanged with the use of the different methods. These implications will be discussed. This study has wider considerations for the measurement of ethnicity in a cohort study, the right of indigenous populations to high quality ethnicity data, and the ongoing critique and development of an epidemiology that is responsive to the needs and aspirations of Maori in New Zealand.

\section{P1-53 COLLIDER-STRATIFICATION BIAS COMPLICATES ESTIMATION OF THE STRENGTH OF RISK FACTORS OF DISEASE RECURRENCE}

doi:10.1136/jech.2011.142976c.46

${ }^{1} \mathrm{~L}$ Smits, ${ }^{* 1,2,3}$ S van Kuijk, ${ }^{2} \mathrm{~L}$ Peeters, ${ }^{1} \mathrm{P}$ Leffers, ${ }^{1,4,5} \mathrm{M}$ Prins, ${ }^{6} \mathrm{~S}$ Sep. ${ }^{1}$ Department of Epidemiology, CAPHRI School for Public Health and Primary Care, Maastricht University Medical Centre, Maastricht, The Netherlands; ${ }^{2}$ Department of Obstetrics, Maastricht University Medical Centre, Maastricht, The Netherlands; ${ }^{3}$ Department of Gynecology, Maastricht University Medical Centre, Maastricht, The Netherlands; ${ }^{4}$ Department of Clinical Epidemiology, Maastricht University Medical Centre, Maastricht, The Netherlands; ${ }^{5}$ Department of Medical Technology Assessment, Maastricht University Medical Centre, Maastricht, The Netherlands; ${ }^{6}$ Department of Internal Medicine, Maastricht University Medical Centre, Maastricht, The Netherlands

Knowledge of factors influencing recurrence risk is essential in the prevention of disease recurrence. In this paper, we show that correct estimation of the strength of such factors is, however, troublesome. We performed a simulation study of the recurrence risk of a fictional pregnancy-related disorder, Y. We assumed that there were four component causes of $\mathrm{Y}, \mathrm{X} 1$ representing the determinant under study, $\mathrm{X} 2$ and $\mathrm{X} 3$ representing unmeasured determinants, and $\mathrm{X} 4$ representing pregnancy as a necessary condition for developing $\mathrm{Y}$. We stipulated that each woman would become pregnant twice. RR of disease during the first pregnancy for $\mathrm{X} 1+$ (presence) vs $\mathrm{X} 1$ (absence) was 19.0. Attributable risk (AR) was 0.18. RR of recurrent disease for $\mathrm{X} 1+$ vs X1- during the second pregnancy, calculated among women with previous disease, was apparently 1.0, and AR was apparently 0.00 . However, we show that real RR and AR were considerably higher (19.0 and 0.95, respectively). Our simulation shows that selection of a study population on the basis of previous disease can lead to underestimation of the strength of recurrence risk factors. The bias involved is a form of collider-stratification bias. We urge for extra caution in the interpretation of studies of recurrence risk factors.

\section{P1-54 EXPOSURE EFFECT ESTIMATES DIFFER SUBSTANTIALLY BETWEEN MARGINAL STRUCTURAL MODELS AND CONVENTIONAL MODELS: RESULTS OF A SYSTEMATIC REVIEW}

doi:10.1136/jech.2011.142976c.47

${ }^{1,2} \mathrm{D}$ Suarez, ${ }^{* 1} \mathrm{R}$ Borràs, ${ }^{3,4} \mathrm{X}$ Basagaña. ${ }^{1}$ Fundació Parc Tauli, Universitat Autònoma de Barcelona, Sabadell, Barcelona, Spain; ${ }^{2}$ Comisión del Mercado de las Telecommunications, Barcelona, Spain; ${ }^{3}$ Centre for Research in Environmental Epidemiology (CREAL), Barcelona, Spain; ${ }^{4}$ MIM-Hospital del Mar, Barcelona, Spain; ${ }^{5}$ CIBERESP, Barcelona, Spain

Introduction Marginal structural models (MSMs) were developed to address time-varying confounding in nonrandomized exposure effect studies. It is unclear how estimates from MSMs to conventional models differ in real settings and how the MSMs are implemented in the literature.

Methods We systematically reviewed the literature of MSMs since 2000 retrieving papers from both PubMed and ISI Web of Knowledge databases.

Results Data to compare MSMs and conventional models were obtained from 65 papers reporting 164 exposure-outcome associations. In $18(11.0 \%)$, the two techniques resulted in estimates with opposite interpretations, and in $58(39.7 \%)$ estimates differed by at 